\title{
La rhétorique de la catastrophe
}

The rhetoric of catastrophe

\section{Yannis Thanassekos}

\section{OpenEdition}

\author{
Journals
}

Édition électronique

URL : http://journals.openedition.org/questionsdecommunication/2273

DOI : 10.4000/questionsdecommunication.2273

ISSN : 2259-8901

\section{Éditeur}

Presses universitaires de Lorraine

\section{Édition imprimée}

Date de publication : 31 décembre 2007

Pagination : 41-56

ISBN : $978-2-86480-849-7$

ISSN : 1633-5961

\section{Référence électronique}

Yannis Thanassekos, "La rhétorique de la catastrophe », Questions de communication [En ligne], 12 2007, mis en ligne le 24 septembre 2015, consulté le 08 avril 2021. URL : http://

journals.openedition.org/questionsdecommunication/2273; DOI : https://doi.org/10.4000/ questionsdecommunication.2273 


\title{
$>$ DOSSIER
}

\author{
YANNIS THANASSEKOS \\ Fondation Auschwitz, Bruxelles \\ info.fr@auschwitz.be
}

\section{LA RHÉTORIQUE DE LA CATASTROPHE}

\begin{abstract}
Résumé. - À la charnière du $X X^{e}$ au $\left.X X\right|^{e}$ siècle s'est mis en place un dispositif intellectuel et mental qui tend à accorder à l'« événement Auschwitz » le statut, sur le plan historiographique, d'une matrice pour une révision et une réévaluation de toute l'histoire de l'occident moderne et, sur le plan mémoriel, celui d'un événement sursignifiant à partir duquel doivent s'élaborer les orientations normatives, politiques et juridiques des sociétés contemporaines. Sans négliger les enjeux historiographiques de ces relectures, cet article s'intéresse surtout à leur rhétorique laquelle, mobilisant le pathos et l'hyperbole, a engendré une doxa fondée sur quelques postulats: «Auschwitz » serait un événement qui échappe à la raison, absolument unique, incomparable et irreprésentable. Ce faisant, l'événement se voit complètement decontextualisé, soustrait à l'histoire, pour devenir un pur objet métaphysique livré à des spéculations théologiques et mystiques. Dans sa radicalité actuelle cette rhétorique semble ainsi recycler, sous le signe de la «dernière catastrophe », tous les schémas de pensée catastrophistes et apocalyptiques propres à toutes les théologies politiques qui n'ont eu de cesse de proclamer l'échec de la raison, l'inanité de l'idéologie du progrès et la fin de tout projet d'amélioration et d'émancipation de l'homme et de la société.
\end{abstract}

Mots clés. - Shoah, Auschwitz, historiographie, événement, révision, rhétorique, catastrophe, pathos, hyperbole, mémoire collective. 
T out donne à penser que le passage du $X X^{e}$ au $\left.X X\right|^{e}$ siècle a mis en place un puissant dispositif intellectuel et mental visant à une relecture de cinq segments historiques qu'on peut tenter de délimiter en s'inspirant des conceptualisations proposées par Fernand Braudel (1969): le premier se rapporte au long terme, il couvre la période qui va du $X V \|^{e}$ au $X X^{e}$ siècle et concerne, pour faire bref, la modernité ; le second se rapporte au moyen terme et couvre la période du $X X^{e}$ siècle ; le troisième relève du court terme et concerne la période 1914-1945; tandis que les quatrième et cinquième segments représentent des « conjonctures » à l'intérieur du segment court et se rapportent respectivement aux périodes 1919-1933 et 1933-1945.

À l'épicentre du dispositif intellectuel qui conditionne la relecture de ces cinq segments se situe l'impétueux retour de ce qui a été jusque là refoulé à la fois sur le plan historiographique et sur le plan de la mémoire collective : l'« événement Auschwitz ». J'entends par «Auschwitz » la métonymie de la criminalité nazie dans sa dimension spécifique de génocide. Double refoulement, dis-je, historiographique et mémoriel. Je rappelle en effet que dans sa longue et victorieuse lutte contre l'histoire événementielle - essentiellement militaire et diplomatique -, l'« histoire monde », l'histoire du long terme, avait fini par refouler, par dévaluer, voire par discréditer entièrement l'« événement» comme n'étant qu'une simple manifestation de surface, quantité négligeable destinée à s'effacer, enjeu passager des passions des acteurs, écume éphémère des grandes lames de fond, des grands mouvements souterrains qui seuls font l'histoire'. Quant au refoulement de cet événement sur le plan de la conscience et de la mémoire collectives, inutile d'y insister, il a été déjà largement commenté et de diverses façons.

Dans ce dispositif de révision historique, l'« événement Auschwitz 》, non seulement réhabilite le très court terme (Mayer, 1988 : 8) - 19421945 -, mais en plus il s'attribue, en tant qu'événement précisément, un double statut. D'une part, sur le plan historiographique, le statut d'une «matrice » à partir de laquelle on scrute, on revisite et on réévalue désormais toute l'histoire de l'Occident moderne; de l'autre, sur le plan de la mémoire collective, le statut d'un événement «sursignifiant » à l'aune duquel doivent désormais s'élaborer les orientations normatives, éthiques, politiques voire juridiques des sociétés contemporaines - l'aspect juridique ne doit pas être sous-

\footnotetext{
Par cette remarque, je ne veux nullement dévaluer l'histoire du long terme ni sa portée. Elle a puissamment contribué à l'approfondissement de nos connaissances historiques tant par l'élargissement du questionnaire que par la diversification des objets historiques.
} 
estimé, ainsi que l'attestent les grands débats actuels sur les lois dites «mémorielles». Pour le dire autrement: le récit de la Shoah se présente, à l'intérieur du dispositif en question, à la fois comme le bilan de l'histoire moderne et comme le récit fondateur des temps post-modernes et, pour certains, des temps post-historiques, posthumanité et post-culturels. Toutefois, ce qui m'intéresse ici, sur le plan aussi bien discursif qu'historiographique, ce ne sont pas les enjeux interprétatifs de ces relectures et de ces révisions, mais exclusivement leur rhétorique. Les enjeux interprétatifs sont certes immenses, mais leur analyse fait appel à un autre type d'enquête qui n'entre pas dans le cadre du présent dossier. II est vrai cependant qu'on ne peut séparer rigoureusement les enjeux interprétatifs des enjeux rhétoriques. Aussi ne signalerai-je les premiers que là où les seconds semblent, en quelque sorte, les surdéterminer.

En anticipant sur les développements qui vont venir, je dirais que la rhétorique de la majeure partie de ces relectures a l'ethos propre à la parole oraculaire et se présente sous le signe de l'hyperbole, du pathos et de la catastrophe. Les moyens de la preuve sont davantage techniques qu'extra-techniques (Aristote). Quant à l'ordre de fonctions, le souci qui prime est celui de movere au détriment de celui de docere (Cicéron). Les enjeux de cette rhétorique se cristallisent sur deux plans : celui de la nomination - plan linguistique -, et celui de la compréhension, par quoi il serait peut-être utile de commencer. Pour anticiper tout malentendu, car mes propos risquent d'être mal compris sinon mal accueillis, je me dois de préciser que ce qui suit ne vise nullement à relativiser ou à minimiser l'importance et la portée du génocide perpétré par le III Reich, loin s'en faut, mais d'explorer certains types de discours qui se construisent à partir et autour de cet événement capital.

Partant et parlant du II-Septembre, Jacques Derrida (2004) grand maître en rhétorique, a fixé la prémisse : il stipule qu'il n'y a d'« événement » à proprement parler, événement digne de ce nom, que lorsque surgissant, ce qui surgit demeure incompréhensible, hors entendement et hors raison. S'il est compris, c'est qu'il est d'emblée entré dans un «programme », dans un « réseau » déjà disponible d'explications, d'interprétations et de nominations, et de ce fait même, il perd irrémédiablement son statut d'événement au sens fort du terme - « événement majeur » (Derrida, Habermas, 2004). La filiation heideggérienne de cette position ne m'intéresse pas ici et elle ne sera donc pas discutée. Je constate en revanche que cette rhétorique n'est pas nouvelle. Son application à l'attentat du I I-Septembre n'est que le transfert vers cette catastrophe d'une rhétorique pour ainsi dire archétypale, déjà constituée et largement diffusée autour, précisément, 
du génocide 2 . En effet, l'archétype de l'« Événement » tel qu'il est posé par Jacques Derrida n'est autre que l'« événement Auschwitz », dans la mesure où depuis une trentaine d'années maintenant, toute une rhétorique a contribué à formater une doxa qui stipule que ce qui s'est produit à Auschwitz est, d'une part radicalement incompréhensible, hors raison et entendement, et de l'autre strictement irreprésentable ${ }^{3}$. Pour ce qui est du montage et du démontage de la rhétorique de l'« irreprésentable » et de l'interdit de la représentation, je ne peux que renvoyer à l'excellent ouvrage de Georges Didi-Huberman, Images malgré tout (2003). Ce qui m'intéresse ici en revanche c'est, dans un premier temps, la rhétorique de l'« incompréhensibilité radicale » de l'événement. Pour faire face à l'objection majeure de Giambattista Vico ( 1710 ; 1744) - selon laquelle « ce qui est fait de mains d'hommes, les hommes peuvent le comprendre »-cette rhétorique s'est efforcée de mettre un bémol à son dogmatisme en introduisant une distinction entre le registre de la question du « comment » et le registre de la question du « pourquoi ». Cette distinction suggère qu'il y aurait en quelque sorte deux types d'événements. L'un qui serait de l'ordre de la pure factualité et qui pourrait par conséquent être visé par la première question, celle du « comment »-par quoi il faut entendre la description, à l'aide de la rationalité scientifique, des structures, des mécanismes et des processus de décision qui l'on produit. Cet ordre d'événements pourrait alors être qualifié, pour reprendre l'expression de Paul Ricœur, d'« infra-signifiant ». Et puis il y aurait, par-delà cette pure factualité, un « autre » événement qui, échappant à la raison, échapperait aussi radicalement à la seconde question

${ }^{2}$ Ce n'est d'ailleurs pas le seul transfert sémantique que l'on peut observer. En effet, un grand nombre de locutions qui ont vu le jour pour évoquer l'« événement Auschwitz » telles que, par exemple, «plus jamais ça », «plus rien ne sera comme avant », « cela dépasse l'entendement », « innommable », « indicible », « exprimer l'inconcevable », 《 rupture », « porter témoignage », « devoir de mémoire » etc., migrent aujourd'hui vers d'autres catastrophes ou traumatismes à portée collective, comme par exemple, sur le plan écologique, les conséquences du naufrage du pétrolier Erika, la terrible catastrophe du tsunami en Indonésie, le traumatisme collectif lié à l'affaire Dutroux en Belgique... - autant d'événements qui ont désormais leurs dates de commémoration. Ce processus migratoire fonctionne dans un double mouvement apparemment paradoxal : tandis que le premier fait craindre une « banalisation » et une « relativisation » du prototype, le second au contraire contribue à sa consécration comme « norme référentielle » par rapport à laquelle doivent se mesurer toutes les autres catastrophes, passées, présentes et futures.

Cette doxa comporte aussi de nombreuses autres variations qu'il est impossible de mentionner ici, comme celle par exemple qui consiste à échafauder l'hypothèse, psychanalytique en l'occurrence, selon laquelle la Shoah serait un «événement-sanstémoin » (voir Felman, 1990). 
celle du «pourquoi » précisément ${ }^{4}$. Ce serait un événement «sursignifiant ». Ici, raison, sciences et explications rationnelles sont irrémédiablement caduques. Nulle réponse possible à la question du « pourquoi » d'Auschwitz. II y aurait, dans l'événement, un « reste » impénétrable par la raison. Sur ce « reste», Giorgio Agamben ( 1999) a construit toute une rhétorique « négative »- au sens de la théologie négative - que je n'examinerai pas dans cet article ${ }^{5}$. Cette disjonction antinomique entre le «comment » et le «pourquoi » peut également prendre une forme rhétorique prescriptive : «On ne peut comprendre le génocide commis par les nazis parce que le comprendre serait une manière de l'approuver : s'il faut donc continuer à recenser et à étudier les faits pour montrer jusqu'où ont pu conduire les mécanismes du Reich nazi, il faut refuser de les comprendre » (Jauss, Le Monde, 06/09/96)6. Cette position est défendue aussi, nous le savons, par Primo Levi (1958; 1986)7. Pour d'autres, le projet même de comprendre le «pourquoi »d'Auschwitz serait de l'ordre d'une obscénité absolue. Ainsi, pour Claude Lanzmann (I990: 279), « il y a bien une obscénité absolue du projet de comprendre $»^{8}$. Vouloir comprendre, chercher à comprendre Auschwitz, serait absolument obscène. Mieux encore, la sentence prend la forme rhétorique que voici : «La radicalité ne se divise pas: pas de pourquoi, mais pas non plus de réponse au pourquoi du refus du pourquoi sous peine de se réinscrire dans l'obscénité à l'instant énoncée » (Lanzmann: ibid.). Bref, j'affirme que la question du «pourquoi » est obscène et si vous osez poser la question «pourquoi est-ce obscène? », alors vous êtes irrémédiablement coupable d'une double obscénité. Ce genre d'affirmations oraculaires mime la vielle rhétorique de

\footnotetext{
${ }^{4}$ La distinction épistémologique entre la question du «comment» et celle du « pourquoi » - la première étant accessible à la connaissance, l'autre non -, participe d'une longue histoire philosophique et théologique. Nous ne pouvons l'aborder ici. Signalons toutefois que depuis le XVIII e siècle, cette thématique constitue l'un des topos majeur de la pensée conservatrice et réactionnaire dans sa lutte contre les Lumières. E. Burke (1757: I7|-|72, souligné par nous) l'a formulé en son temps de façon claire : «Comment notre industrie, quelles qu'en soient les ressources, pourraitelle jamais débrouiller la grande chaîne de causes dont les maillons remontent jusqu'au trône de Dieu lui-même? Dès que nous perdons de vue les qualités immédiatement sensibles des choses, nous sortons de notre sphère. Tous les efforts que nous faisons ensuite sont vains et témoignent de ce que nous nous trouvons dans un élément qui n'est pas nôtre [...]. Si j'avais à expliquer le mouvement d'un corps qui tombe, je dirais qu'il est causé par la gravité, et j'entreprendrais de montrer ensuite comment agit cette force, sans essayer de montrer pourquoi elle agit ainsi ».

5 Pour une judicieuse critique de G. Agamben, voir Mesnard, Kahan (2002).

6 Rappelons que H. R. Jauss fut un engagé volontaire à la Waffen-SS, capitaine de la Division Charlemagne et décoré de la Croix d'Or en 1944.

7 Pour une analyse critique de cette position de P. Levi, voir Thanassekos (2006).

8 Cette thèse n'est pas défendue que par Cl. Lanzmann, toute une nébuleuse l'a fait sienne.
} 
l'antinomique d'après laquelle l'unité de contraires nous conduit à une unité prétendument supérieure.

Cette rhétorique d'une « radicalité qui ne se divise pas », se complète par une deuxième prise de position qui stipule, dans une logique immanente à la première, que l'« événement Auschwitz » est singulier et unique. Épistémologiquement parlant, cette affirmation n'a que la valeur d'une pure tautologie. Discutant avec un physicien et voulant lui démontrer que la seule discipline scientifique capable d'énoncer des certitudes c'était bien la discipline historique - contrairement précisément aux prétentions naturalistes de l'inductionnisme -, un historien déclara ceci audit physicien : «Philippe le Bel traversa ce pont le 18 février de l'an 1308 et puis-je vous affirmer avec une certitude absolument absolue qu'il ne le traversera plus jamais ». La boutade énonce une évidence : tout événement historique est par définition singulier et unique - ce qui, loin d'exclure des comparaisons et des mises en rapport, les rend au contraire nécessaires puisque, privée de la possibilité d'expériences, la discipline historique n'a d'autres voies pour produire de l'intelligibilité que celle de la méthode comparative qui ouvre la possibilité à des généralisations - si elle ne veut pas bien entendu se réduire à une simple production documentaire. Ici aussi cependant, comme précédemment, la doxa de l'incompréhensibilité radicale tente de contourner l'objection mais au prix d'une curieuse « hyperbolisation »: oui, nous disent-ils, tout événement est singulier, mais cela ne peut concerner que les événements « infrasignifiants 》. Or, en l'occurrence, poursuivent-ils, nous sommes en présence d'un événement «sursignifiant » ce qui permet de conclure qu'il est non seulement singulier et unique, mais absolument singulier et absolument unique et donc, absolument incomparable - un événement qui ne peut être comparé à nul autre. Triple interdit donc : pas de « pourquoi », pas de « comparaison », pas de « représentation ».

Pour résumer, nous sommes donc en présence d'une doxa construite autour de quatre «lieux» (topoi). L'événement «Shoah» serait: irréductible à la raison et aux explications rationnelles ; irreprésentable ; absolument singulier et absolument unique et, partant, absolument incomparable. Toute tentative de discuter ou de mettre en question l'une de ces quatre prémisses nous rendrait irrémédiablement suspects de vouloir, au mieux, relativiser l'événement et sa portée. Inutile de préciser qu'avec de tels présupposés, l'événement se trouve littéralement projeté hors Histoire, expulsé du domaine du connaissable, pour devenir un objet purement métaphysique et métahistorique, livré aux spéculations mystico-religieuses les plus diverses, ce dont ne se prive nullement une certaine littérature. Quant aux problèmes que soulève la transmission de cette expérience historique, cette même rhétorique fait 
feu de tout bois : « [...] nulle intelligibilité, c'est-à-dire nul savoir vrai, ne préexiste à la transmission » (Lanzmann, 1990 : 279). Traduction : tout enseignement en la matière ne peut être que strictement initiatique. Allez faire de la pédagogie avec cela!

Passablement terroriste, cette rhétorique à l'ethos oraculaire vire au pathos lorsqu'elle affronte le problème de la nomination de l'événement ainsi configuré - entendu, bien évidemment, que toute nomination est aussi attribution de sens. Je dois beaucoup dans ce qui suit à l'excellent article sur cette même question de Michael Bernstein paru dans la revue Le débat en 1998. En effet, à lire les nombreux théoriciens en la matière, l'« événement Auschwitz » apparaît comme un novum radical ou, pour reprendre une formule connue de François Lyotard dont l'effet rhétorique est sans pareil, comme « un séisme d'une magnitude telle qu'elle aurait détruit les instruments capables de le mesurer ». Bref, un événement qui aurait signifié la faillite totale et irrémédiable non seulement de nos techniques de mesure, mais aussi de nos catégories explicatives du monde, et qui aurait ruiné par conséquent la stabilité de notre compréhension de l'histoire et de nous-mêmes. Cette thèse hyperbolique, qui a acquis une autorité presque irrécusable aujourd'hui, entraîne un double mouvement simultané et paradoxal mis en évidence par Michael Bernstein. D'une part, l'aveu explicite que notre langage et notre terminologie disponibles seraient incapables de nous fournir un discours tant soit peu à la mesure ou plutôt à la démesure de l'événement et, de l'autre, en contrepoint, un effort obstiné pour forger un vocabulaire nouveau susceptible de fournir une cohérence au discours proposé. Et comme il fallait s'y attendre, plus I'on déclare irrémédiable l'échec de la pensée confrontée à l'événement, plus obstinée est la recherche d'un terme, d'un vocabulaire nouveau, censé remédier tant soit peu à cette carence de représentation conceptuelle. Il en résulte un pathos linguistique orné d'appellations les plus diverses. En voici un petit échantillon retenu par Michael Bernstein : « mysterium tremendum », «crime ontologique », « abomination métaphysique », «Chose sans nom », « La Chose », « Chose » (tout court), « novum », « différend », « une rupture du tissu de l'existence », « après Auschwitz une nouvelle forme de connaissance investit l'esprit », «les camps de la mort sont une réalité qui [...] oblitère la pensée et le programme humain de réflexion » ou encore «Le sens d'Auschwitz c'est de ne pas avoir de sens $\rangle^{9}$, et on pourrait en ajouter d'autres... Situation pathétique, marquée à la fois par une défense tortueuse et longue de la légitimité des énoncés proposés et par un aveu d'impuissance

9 E. Levinas, cité par B. Frappat «Entretien avec Emmanuel Levinas » (Le Monde, 14/03/94). 
devenu quasi rituel en la matière. Par de longs plaidoyers en faveur d'un nouveau lexique pour « l'écriture du désastre » et, en même temps, par l'affirmation selon laquelle toute mise en concept ne peut que trahir l'immensité du cataclysme. On observe le même paradoxe apparent chez tous ceux qui postulent que seul le « silence absolu » convient face à l'immensité de l'événement alors même qu'ils se complaisent, à titre de démonstration de leur postulat, dans un assourdissant bruit linguistique et même médiatique. Silence et logorrhée sont en fait les deux faces des pièces que contient l'archive de la mystique.

En l'espèce, le pathos n'est pas seulement l'expression d'une coquetterie linguistique, il devient l'outil par excellence pour faire barrage à toute évaluation rationnelle des contenus de pensée proposés. II est intéressant de signaler les termes dans lesquels Claude Lanzmann tenta d'expliquer et de justifier son choix du terme «Shoah » comme titre de son œuvre filmique. Je le cite : «La vérité est qu'il n'y avait pas de nom pour ce que je n'osais même pas alors l'appeler l'"événement" [...]. Pour moi "Shoah" était un signifiant sans signifié, une profération brève, opaque, un mot impénétrable, infracassable, comme un noyau atomique $\rangle^{10}$. II restitue ensuite sa conversation à cet égard avec l'un de ses partenaires qui lui demandait qu'est-ce que « Shoah » veut dire : « Je ne sais pas, cela veut dire "Shoah" »-« Mais il faudra traduire, personne ne comprendra » lui rétorque son interlocuteur ; - « c'est précisément ce que je veux, que personne ne comprenne » réplique Claude Lanzmann. Ici aussi, comme précédemment avec l'obscénité du « pourquoi », la radicalité ne se divise pas. À événement incompréhensible, nomination incompréhensible". Et c'est ainsi qu'une

\footnotetext{
${ }^{10} \mathrm{Cl}$. Lanzmann, « Ce mot de "Shoah"» (Le Monde, 26/02/05 : p. 14).

1 " J'aimerais ouvrir ici une brève parenthèse. Cette façon de penser l'événement et de le mettre en langage contraste de façon remarquable avec l'extrême simplicité et la modestie qui caractérisent tous les témoignages des survivants, plus particulièrement de ceux dont le récit, fort élaboré et rigoureux, constitue pour nous une référence quasi incontournable. II y a là une sorte d'énigme. On pourrait certes objecter que ceux qui ont vécu cette expérience peuvent mieux l'exprimer en des termes simples et presque prosaïques sans pour autant trahir la profondeur de la rupture subie et que, en revanche, pour ceux qui ne l'ont pas vécue, tout effort de représentation s'abîme au regard de cette insondable rupture. Pourtant, une telle réponse laisse à désirer. Les témoins dont nous parlions, de D. Rousset à J. Semprun en passant par P. Levi, J.Amery, C. Delbo et G.Tillion pour n'en citer que quelques-uns - ont tous mis l'accent sur le caractère inimaginable de l'expérience vécue, ainsi que sur l'indigence du langage et de nos catégories coutumières pour en rendre compte. Pourtant, ils ont réussi, avec une clarté qui force l'admiration, à faire usage de notre langage le plus courant pour entrer en communication avec nous et faire comprendre, presque de l'intérieur, leur vécu. Et il faut insister sur le fait qu'il ne s'agit pas ici de simples récits, mais de véritables élaborations en profondeur à la fois du vécu lui-même et de sa signification anthropologique et philosophique. Voir à ce sujet le précieux chapitre IV, « Communiquer », de l'ouvrage posthume de P. Levi, Les naufragés et les rescapés. Pour ce qui est du langage chez P. Levi en rapport avec une critique judicieuse des courants actuels (dont G. Steiner), voir Fr. Rastier (2005).
} 
œuvre, filmique en l'occurrence, a fini par remplacer l'événement lui-même. À la limite et à la faveur d'une campagne médiatique soutenue, ce qui fait désormais événement - tout au moins dans certains milieux - ce n'est plus l'événement historique lui-même mais Shoah, le film².

Cette surenchère des néologismes surchargés de connotations théologiques, métaphysiques et mystiques résulte incontestablement de la double prémisse que nous avons évoquée: celle de la nature radicalement incompréhensible de l'événement d'une part, celle de sa singularité absolue et radicale de l'autre. Comment faut-il interpréter toute cette rhétorique qui n'est en fait qu'une mise en scène de soi ? Comment faut-il interpréter ses tropes qui sont autant de discours autoréférentiels et dont les énoncés, si peu crédibles sur le plan du raisonnement historique, ont fini par acquérir sur le plan de l'opinion commune la force d'une évidence? La raison en est simple : ils font précisément l'impasse au raisonnement et à la discussion rationnelle au seul profit du pathos qui constitue un horizon d'attente fortement répandu de nos jours. II ne s'agit plus d'arguments; il s'agit d'énoncés pathétiques qui ne s'adressent même pas aux sentiments mais au pathos du sublime, de l'extase et de l'horreur. Le locuteur parle en fait à lui-même. Sur le plan formel, cette rhétorique vise d'abord à intimider, voire à culpabiliser l'intelligence, dès lors qu'elle s'efforce de comprendre, en même temps qu'elle fait signe à une vieille stratégie rhétorique de nature sacerdotale laquelle, hier comme aujourd'hui, cherche à tirer les dividendes des « énigmes » supposées irrésolues, des «mystères 》 déclarés impénétrables et des « volontés 》 posées comme insondables. Quant au fond, le problème est tout autre - et plus important me semble-t-il. En effet, à la suite de Michael Bernstein (1998), je crois que par-delà le pathos et les hyperboles dont elle use et abuse pour qualifier l'événement, cette littérature trouve son origine moins dans l'événement lui-même que dans un univers intellectuel et mental qui lui est largement antérieur et extérieur. En effet, depuis plus d'un siècle maintenant et singulièrement à partir de la Grande Guerre, s'est formée en Europe une culture, aux visages multiples, qui a fait de l'horreur apocalyptique sa figure centrale, son motif majeur. Cette culture elle-même ne faisait que prolonger, en la radicalisant dans l'entre-deux-guerres, la nébuleuse idéologique du pessimisme culturel et du décadentisme qu'avaient mise en branle les premières réactions contre la modernité tout au long d'un $\mathrm{XI} \mathrm{X}^{\mathrm{e}}$ siècle décidément trop long - de 1789 à 1914. On trouve des traces récurrentes de cette «culture catastrophiste $\gg$, de cette vision apocalyptique de l'histoire dans nombre

\footnotetext{
${ }^{12}$ Cette remarque ne tient pas lieu de critique du film Shoah dont je ne sous-estime nullement l'apport historique et mémoriel et encore moins ses qualités intrinsèques.
} 
de discours et de théories dont la conclusion est tout autant lapidaire qu'irrévocable: loin de pacifier la société, le processus civilisationnel n'aurait fait que révéler l'irréductibilité des instincts primitifs, des pulsions de mort et du mal radical qui habitent l'homme, et signer par là même la fin de toute perspective d'amélioration de l'homme et de la société. Perfectibilité et progrès se seraient avérés des croyances naïves, voire aveugles. Kant et Norbert Élias - pour ne citer que ces deux penseurs dans l'espace de l'optimisme raisonné - se seraient lourdement trompés. Les catastrophes historiques sont ainsi pensées comme autant de vengeances apocalyptiques des instincts primitifs contre une civilisation rationnelle avérée incapable de les dompter. Sous cet angle, la rhétorique sur la «Shoah » se présente, dans sa radicalité actuelle, comme la confirmation ultime, la preuve irréfutable, du diagnostic depuis longtemps posé sur le destin du processus civilisationnel, à savoir l'échec de la Raison, l'apocalypse programmée, la fin des temps, du temps historique en particulier. À proprement parler, il n'y a donc pas de novum, mais la poursuite et le recyclage d'un très vieux discours porté à son paroxysme. Aussi le «trou noir d'Auschwitz » devient-il le creuset dans lequel viennent se fondre en un seul tenant et prendre forme unitaire, toutes les intuitions, toutes les appréhensions, tous les sentiments et tous les schémas de pensées d'ordre apocalyptique qui n'ont pas manqué de faire éruption de manière récurrente dans l'histoire de l'Occident. Le grand romancier américain Norman Mailer a formulé de façon saisissante cette perspective. Selon lui, «la théorie qui nous aide à comprendre le monde [...] tient en un seul mot :"catastrophe". J'appartiens à l'école catastrophiste de l'histoire $>^{13}$.

Le plus souvent, les raisons avancées pour expliquer le retard pris dans l'évocation de la Shoah comme événement central de l'histoire du $X X^{e}$ siècle sont d'ordre à la fois psychologique et politique. Ce retard s'expliquerait par le fait qu'il nous a fallu du temps, d'une part, pour surmonter le traumatisme et pour prendre pleinement conscience des dimensions du désastre - autrement dit qu'il a fallu du temps pour élaborer l'événement et vaincre son refoulement - et, de l'autre, pour faire sauter l'occultation qu'aurait subie la mémoire juive du désastre par la toute puissante mémoire de la Résistance dans les premières décennies après la Libération. Les développements qui précèdent invitent à penser que cette explication est moins évidente et assurée qu'elle n'apparaît à première vue. En tout cas, la part de vérité qu'elle contient ne doit pas occulter à son tour d'autres aspects du phénomène. Comme l'a souligné Michael Bernstein (1998), contrairement à une

\footnotetext{
${ }^{13}$ Blumenfeld S., « Entretien avec Norman Mailer (à l'occasion de la publication du premier volume de son nouveau roman, Un château en forêt) », Le Monde 2 (04/08/07, pp. 12- 13).
} 
certaine vulgate, les témoignages et les récits qui ont rendu publiques les dimensions et la portée des crimes du national-socialisme, génocide compris, ont été finalement assez précoces - dès les premières années de la Libération - et ont connu immédiatement une large diffusion ainsi qu'une reconnaissance incontestée par l'attribution notamment des prestigieux prix. Des œuvres - témoignages ou romans - comme celles de David Rousset (1946), de Robert Antelme (1947), de Roger Ikor (1955) et d'André Schwartz-Bart (1959), connurent en effet un impact considérable et occupèrent une place notable dans le paysage culturel des deux premières décennies après la Libération. II se peut alors que s'il a fallu du temps pour parler de la Shoah dans les termes d'aujourd'hui, ce ne fut pas seulement ou exclusivement pour les raisons évoquées mais parce qu'il aura fallu du temps aussi - une bonne trentaine d'années pour qu'émerge et se consolide un contexte idéologique susceptible d'accueillir un tel discours, le sanctionner et le consacrer ${ }^{14}$.

Ce processus impliquait une triple opération étalée dans le temps. La première est signalée par Michael Bernstein : il a fallu d'abord « déloger », sur le plan des nos représentations, ce qui, dans la grande série des cataclysmes historiques, pourrait contester à la Shoah la place qu'elle occupe à présent dans notre univers mental - à commencer par le premier carnage international vécu par les contemporains comme un véritable cataclysme, comme une vraie rupture. J'ajouterai deux opérations supplémentaires. Celle qui consista à dé-contextualiser radicalement l'événement, à l'extraire de l'histoire pour lui conférer le statut d'une catégorie supra-historique ou plutôt anhistorique. Celle aussi qui, dans la foulée, visa à consacrer et légitimer comme allant de soi une lecture anachronique et univoque de l'histoire, c'est-à-dire une lecture sous le seul prisme du « désastre » devenue une catégorie méta-historique fondamentale. La relecture politique de tous les segments historiques, évoqués en introduction se métamorphose ainsi en une lecture politique de l'Apocalypse et, par là-même, elle renoue

\footnotetext{
${ }^{14}$ Parmi les nombreux symptômes de ce contexte idéologique il y aurait lieu de signaler, à titre indicatif, le fait que depuis le début des années 70, c'est-à-dire depuis qu'apparait ce discours hyperbolique sur la Shoah, s'enfle aussi, surtout aux États-Unis, toute une mentalité collective - nourrie d'une vieille tradition évangéliste -, fascinée par l'attente de la catastrophe à venir. La production cinématographique et télévisuelle des années 70 à nos jours est, en la matière, un indicateur significatif. II serait intéressant d'analyser par exemple de façon plus précise des films américains tels que Independance Day (Spielberg, 1996). La double thématique de la catastrophe et du survivant qui empreigne l'univers mental américain - ce film est typique sous ce rapport - explique peut-être aussi l'extraordinaire identification des américains avec le génocide et la place qu'occupe la Shoah comme référence dans la politique des États-Unis - ce qui constitue aussi une façon de colmater la culpabilité collective par rapport au génocide des amérindiens. Voir à ce sujet l'article de J.-M. Dreyfus (2004).
} 
avec la vieille tradition de la théologie politique. Réduisant sans plus la modernité à sa seule dimension de «rationalité instrumentale » et la politique moderne à sa seule composante de «rationalité bureaucratique », assimilant abusivement les principes cognitifs de la science aux applications perverses des techniques, le diagnostique posé par ce type de lecture découle de sources: dans son article significativement titré, «Pensez l'apocalypse », Maurice Blanchot ${ }^{15}$ fait sienne la formule qu'il attribue au poète Paul Celan d'après laquelle « la Shoah est, face à l'Occident, la révélation de son essence ». C'est une vieille rengaine, mille fois répétée lors de «l'affaire Heidegger» notamment: «Dans l'apocalypse d'Auschwitz ce n'est ni plus ni moins que l'Occident, en son essence, qui s'est révélé [... . 》 (Lacoue-Labarthe, 1988 : 59), ou encore, « C'est pourquoi cet événement, l'Extermination, est à l'égard de l'Occident, la terrible révélation de son essence » (ibid. : 63). Autrement dit, la modernité est responsable du génocide ou plus radicalement, nous précise le sociologue polonais Zygmut Baumann (1989), rien dans la modernité n'était de nature à empêcher que l'holocauste s'accomplisse ${ }^{16}$.

Je ne discuterai pas dans ces pages de la pertinence ou non de ce type de thèses qui sont au cœur des problèmes interprétatifs, je constate simplement que, du point de vue rhétorique, une telle position équivaut à une relecture grossièrement anachronique et univoque de la modernité, une relecture qui évacue purement et simplement, au profit de la seule catastrophe, tous les autres aspects et dimensions de la modernité, notamment ses aspects émancipateurs, ses acquis, ses autres potentialités non avérées, les espoirs et les promesses qu'elle a nourris et qu'elle pourrait encore continuer à nourrir. Ceci est encore plus significatif si l'on examine la double conjoncture 1914-1933 et 19331945 que ladite rhétorique a regroupée sous l'appellation commode de « seconde guerre deTrente ans », ou encore le segment moyen de 1914 à 1989, appelé aussi le « Court XX ${ }^{\mathrm{e}}$ siècle », baptisé par les uns « L'âge des catastrophes », par d'autres, « À l'épreuve du désastre » et par certains autres, «L'ère de l'épouvante » ou encore l'« Europe. Une passion génocidaire $\rangle^{17}$. Autrement dit, un siècle d'une guerre ininterrompue de 75 ans : la Grande Guerre, la guerre d'Espagne, la Seconde Guerre mondiale, les guerres coloniales et enfin la guerre froide ! Le tableau est saisissant, propice à ce type de rhétorique qui n'a que deux mots :violence

\footnotetext{
${ }_{16}^{15}$ M. Blanchot, « Heidegger et la pensée nazie », Le Nouvel Observateur (22-28/0 I/88).

${ }^{16}$ Je me permets à cet égard de renvoyer à ma critique de l'ouvrage du sociologue polonais, «Politique génocidaire et modernité. À propos de l'ouvrage de Z. Baumann [1989]» (Thanassekos, 2003).

${ }^{17}$ Ce n'est là qu'un petit échantillon des titres de quelques ouvrages parus ces dernières années.
} 
et barbarie. Une série de concepts l'alimentent: « culture de guerre », « Guerre civile européenne », «brutalisation », « franchissement de seuils de violence », « déshumanisation de l'adversaire », « retour de l'homme chasseur », « régression de seuils d'inhibition », « retour à l'horde sauvage $»$, etc. ${ }^{18}$ Le fait que la constellation des auteurs qui proposent aujourd'hui ce type de relecture de l'histoire soit multiforme et aux présupposés souvent contradictoires, n'atténue nullement le sens unilatéral de l'approche. Tout se passe comme si ces différents segments et conjonctures n'étaient que des volumes à ranger dans la grande bibliothèque des catastrophes, chaque volume n'étant qu'une sorte d'introduction au suivant jusqu'au dernier volume, celui de la Shoah. C'est une extraordinaire perversion de la thèse selon laquelle toute histoire est une histoire contemporaine. Même chez Benedetto Croce (1920) - à qui on doit cette formule -, le «présentisme » ne donne licence à une telle lecture grossièrement anachronique de l'histoire. Dans son fameux plaidoyer pour une historisation du national-socialisme, Martin Broszat (1990a ; 1990b) avait raison de nous mettre en garde contre une telle tendance qui finissait par ne voir dans l'histoire allemande, depuis Luther, qu'une série d'étapes qui menaient inexorablement au nazisme et à Auschwitz - une sorte de radicalisation à l'extrême de la théorie sur « la voie spécifiquement allemande » de modernisation. De façon significative une telle relecture unilatérale du court $X X^{e}$ siècle devenu «l'ère de la parfaite culpabilité », fait l'impasse sur une série de questions historiographiques et occulte des pans entiers de notre histoire récente. II faudrait un autre article pour illustrer les ravages de ce type de révision appliquée à la Grande Guerre, à la Révolution russe, à la République de Weimar, voire à I'histoire du III Reich ${ }^{19}$.

\section{Conclusion}

Brièvement, j'évoquerai le fait que, selon ce type de relecture, le bilan de la Seconde Guerre mondiale se résume en deux mots: violence et barbarie. Ce faisant, on finit par évacuer purement et simplement l'extraordinaire mobilisation de ressources qui fut nécessaire, aussi bien sur le plan militaire que sur le plan social et politique, pour vaincre et écraser le Béhémoth nazi. Plus fondamentalement encore, on néglige, ou on discrédite comme relevant du mythe, la signification et la portée de la Résistance à un double niveau : relativement à la part qu'elle a

\footnotetext{
${ }_{19}^{18}$ Ce pessimisme anthropologique est illustré, entre autres auteurs, parW. Sofsky ( 1996).

${ }^{19}$ Voir une illustration de cet état d'esprit, dans une veine noltienne il est vrai « dégradée », dans la plupart des contributions de l'ouvrage collectif publié sous la direction de S. Courtois (2006).
} 
prise dans la défaite du fascisme et du national-socialisme, ainsi que dans la mise en œuvre des immenses conquêtes sociales et politiques de l'après-guerre devenues la cible d'une politique de démantèlement, politique dont, paradoxalement, la parure éthique se montre très sensible à la question d'Auschwitz. On oublie souvent que le modèle social dit de l'État providence, tant décrié et combattu aujourd'hui, résulte précisément de cette mobilisation de forces sociales instruites par la guerre et la Résistance. Enfin, dans cette même relecture du siècle passé, on fait l'impasse également sur toutes les luttes anticoloniales et les mouvements de libération nationale qu'elles ont animés.

Par ces quelques observations je ne cherche nullement à minorer la profondeur des désastres de ce siècle de violences et de totalitarismes. De même, je ne sous-évalue nullement ni l'effondrement des espoirs associés aux échecs des «utopies positives» qui ont tourné au cauchemar, ni l'ampleur des désillusions amères que ces échecs ont générées. Les promesses non tenues de la modernité obligent à coup sûr à se garder de tout optimisme ; il n'empêche qu'une telle relecture unilatérale de l'histoire sous le signe de la seule catastrophe non seulement lui enlève toute sa complexité, mais en plus accrédite et légitime, à partir d'Auschwitz, le discours dominant selon laquelle les totalitarismes du $X X^{e}$ siècle auraient signé la fin de toute promesse d'émancipation sociale et ramené la politique à son véritable socle présumé, à savoir la seule gestion des intérêts des communautés humaines.

\section{Références}

Agamben G., 1999, Ce qui reste d'Auschwitz, trad. de l'italien par P. Alferi, Paris, Éd. Rivages, 2003.

Antelme R., 1947, L'Espèce humaine, Paris, Gallimard.

Aristote 329-323 av. J.-C., Rhétorique, trad. du grec par M. Dufour et A. Wartelle, Paris, Gallimard, 2003.

Baumann Z., 1989, Modernité et holocauste, trad. de l'anglais par P. Guivarch, Paris, Éd. La Fabrique, 2002.

Bernstein M., 1998, «Hommage à l'extrême. La Shoah et l'hyperbole de la catastrophe », Le Débat, I0 I, pp. I83-192.

Braudel F., 1969, Écrits sur l'Histoire, Paris, Flammarion.

Broszat M., 1990a, «Plaidoyer pour une historisation du national-socialisme », Bulletin trimestriel de la Fondation Auschwitz, 24, pp. 27-42.

_l990b, «Échange de lettres avec Saul Friedländer », Bulletin trimestriel de la Fondation Auschwitz, 24, pp. 43-86. 
Burke E., 1757, Recherche philosophique sur l'origine de nos idées du sublime et du beau, avant-propos, trad. de l'anglais et et notes par B. Saint Girons, Paris, Vrin, 1990.

Ciceron, 55 av. J.-C., L'Orateur, trad. du latin par E. Courbaud, Paris, Éd. Les Belles Lettres, 2003.

Courtois S., dir., 2006, Les logiques totalitaires en Europe, Monaco, Éd. du Rocher.

Croce B., 1920, Théorie et histoire de l'historiographie, trad. de l'italien par A. Dufour, Genève, Droz, 1968.

Derrida J., Habermas J., 2004, Le « concept » du II septembre. Dialogues à New York, octobre-décembre 2001 avec Giovanna Borradori, Paris, Galilée.

Didi-Hubermann G., 2003, Images malgré tout, Paris, Éd. de Minuit.

Dreyfus J.-M., 2004, « Comment l'Amérique s'est identifiée à la Shoah », Le Débat, I30, pp. 31-43.

Felman S., 1990, « À l'âge du témoignage : Shoah de Claude Lanzmann », pp. 55145, in : Cuau B., Deguy M. et al., Au sujet de Shoah. Le film de Claude Lanzmann, Paris, Belin.

Ikor R., 1955, Les eaux mêlées, Paris, A. Michel.

Lacoue-Labarthe Ph., 1988, La fiction du politique, Paris, Bourgois.

Lanzmann Cl., 1990, « Hier ist kein Warum », in : Cuau B., Deguy M. et al., Au sujet de Shoah. Le film de Claude Lanzmann, Paris, Belin. Article paru initialement dans la revue Nouvelle revue de psychanalyse, « Le Mal », 38, 1988.

- 2005-2006, « Discours de souvenir prononcé le 9 octobre 2005 au Mémorial de la Shoah à Paris », Les temps modernes, 635-636, pp. 7-8.

Levi P., 1958, Si c'est un homme, trad. de l'italien par M. Schruoffeneger, Paris, Julliard, 1987.

- 1986, Les naufragés et les rescapés. Quarante ans après Auschwitz, trad. de I'italien par A. Maugé, Paris, Gallimard, 1989.

— 2002, L'Asymétrie et la vie articles et essais 1955-1987, trad. de l'italien par N. Bauer, Paris, Laffont, 2004.

Mayer A., 1988, La « solution finale » dans l'histoire, trad. de l'américain par M.-G. et J. Carlier, Paris, Éd. La Découverte, 1990.

Mesnard P., Kahan C., 2002, Giorgio Agamben à l'épreuve d'Auschwitz, Paris, Kimé.

Rastier Fr., 2005, Ulysse à Auschwitz. Primo Levi, le survivant, Paris. Éd. du Cerf.

Rousset D., 1946, Le Système concentrationnaire, Paris, Éd. du Pavois.

Sofsky W., 1996, Traité de la violence, trad. de l'allemand par B. Lortholary, Paris, Gallimard, 1998.

Thanassekos Y., 2003, « Politique génocidaire et modernité. À propos de l'ouvrage de Zygmut Baumann », Bulletin trimestriel de la Fondation Auschwitz, 80-81, pp. |47- 156.

- 2006, «L'œurre de Primo Levi : une réception consensuelle?», Colloque international La réception de l'œuvre de Primo Levi en Europe, aux États-Unis, au 
Canada, en Amérique Latine et en Israël, Bruxelles, 12-14 oct., actes à paraître, Paris, Éd. Kimé, 2008.

Vico G, 1710, L'Antique sagesse de l'Italie, trad. du latin par Br. Pinchard, Paris, Flammarion, 1993.

— 1744, Principes d'une science nouvelle relative à la nature commune des nations, trad. de l'italien par A. Pons, Paris, Fayard, 200 I. 